\title{
MINIMUM BER BLOCK PRECODERS FOR ZERO-FORCING EQUALIZATION
}

\author{
Yanwu Ding, Timothy N. Davidson, Jian-Kang Zhang, Zhi-Quan Luo and K. Max Wong \\ Department of Electrical and Computer Engineering \\ McMaster University \\ Hamilton, Ontario, Canada, L8S 4K1
}

\begin{abstract}
In this paper we derive an analytic expression for the linear precoder which minimizes the bit error rate (BER) for block transmission systems with zero-forcing equalization and threshold detection. The design is developed for the two standard schemes for eliminating inter-block interference; viz, zero padding (ZP) and cyclic prefix $(\mathrm{CP})$. The $\mathrm{CP}$ minimum BER precoder has a structure similar to that of the conventional water-filling discrete multitone (DMT) modulation scheme, but the diagonal water-filling power loading matrix is replaced by a full matrix consisting of a diagonal minimum mean square error (MMSE) power loading matrix postmultiplied by a Discrete Fourier Transform (DFT) matrix. The ZP minimum BER precoder has a corresponding structure. Performance evaluations indicate that the signal-to-noise ratio (SNR) gain of the $\mathrm{ZP}$ and $\mathrm{CP}$ minimum BER precoders over conventional water-filling DMT, MMSE, and orthogonal frequency division multiplexing (OFDM) schemes can be as much as several decibels.
\end{abstract}

\section{INTRODUCTION}

In the transmission of digital data over dispersive media, channel induced inter-symbol interference (ISI) is a major performance limiting factor. To mitigate such an effect, it is often helpful to transmit information-bearing data in equal-size blocks. Examples of block based communication systems include multicarrier systems such as orthogonal frequency division multiplexing (OFDM) [4] and discrete multitone (DMT) modulation [1]. Recently, a broad class of linear block-by-block transmission schemes has been studied in detail $[7,8]$. The block based linear transmitter which maximizes the achievable information rate was derived in [7], and the transmitters which minimize the mean square error of the equalized symbols for both zero-forcing and minimum mean square error (MMSE) equalization were derived in [8]. While the design of a transmitter based on the MMSE criterion is mathematically tractable and results in a precoder that performs reasonably well in practice, such a criterion does not guarantee minimum bit error rate (BER). In this paper, we consider the design of minimum BER linear precoders for systems with zero-forcing equalization and threshold detection.

\section{BLOCK TRANSMISSION}

We employ the generalized block-by-block transceiver model developed in [8], and illustrated in Fig. 1. In this model, for each block of $M$ data symbols $\boldsymbol{s}[n]$, a block of $P$ symbols $\boldsymbol{u}[n]=$ $\boldsymbol{F}_{0} \boldsymbol{s}[n]$ is transmitted across the channel. (The components of these blocks are shown in Fig. 1.) At the receiver, the block of $M$ equalized data symbols $\hat{\boldsymbol{s}}[n]$ is constructed from a block of $P$ received symbols, $\boldsymbol{r}[n]$, using the equalizer $\boldsymbol{G}_{0}, \hat{\boldsymbol{s}}[n]=\boldsymbol{G}_{0} \boldsymbol{r}[n]$. If we choose the data block size $M$ to be greater than the channel order, $L$, and if we choose the transmitted block size $P \geq M+L$, then the inter-block interference (IBI) in $\hat{\boldsymbol{s}}[n]$ is due only to the previous transmitted block, $\boldsymbol{s}[n-1]$. In that case, IBI can be eliminated by adopting zero padded (ZP) transmission, or by transmitting a cyclic prefix (CP) which is removed at the receiver. (For simplicity, we will focus on the case where $P=M+L$.) In the $\mathrm{ZP}$ case, the precoder $\boldsymbol{F}_{0}$ has the form $\left[\begin{array}{c}\boldsymbol{F} \\ \mathbf{0}\end{array}\right]$, where $\boldsymbol{F}$ is $M \times M$ and the equalizer matrix $\boldsymbol{G}_{0}=\boldsymbol{G}$ is $M \times P,[7,8]$. In the CP case, $\boldsymbol{F}_{0}$ has the form $\left[\begin{array}{c}{\left[\mathbf{0}, \boldsymbol{I}_{L}\right]} \\ \boldsymbol{I}_{M}\end{array}\right] \boldsymbol{F}$, where $\boldsymbol{F}$ is $M \times M$ and $\boldsymbol{G}_{0}$ has the form $[\mathbf{0}, \boldsymbol{G}]$, where $\boldsymbol{G}$ is $M \times M,[8,9]$. The operation of both these schemes can be expressed as,

$$
\hat{\boldsymbol{s}}=\boldsymbol{G H F \boldsymbol { H }}+\boldsymbol{G} \boldsymbol{v},
$$

where $\boldsymbol{v}$ is a vector of samples of the additive noise at the receiver. If $\mathrm{ZP}$ transmission is used, $\boldsymbol{H}$ is a $P \times M$ tall Toeplitz matrix, and if $\mathrm{CP}$ transmission is used, $\boldsymbol{H}$ is an $M \times M$ circulant matrix. Equation (1) forms the basis for the ensuing development of the paper. We assume that the channel is known, and that the transmitted symbols are equi-probable binary phase shift keying (BPSK) symbols that are uncorrelated with each other; i.e. $E\left\{s \boldsymbol{s}^{H}\right\}=\boldsymbol{I}$. (An extension to quadrature phase shift keying [QPSK] appears in [3].) We will also assume that the receiver noise is zero-mean, white, and Gaussian, with covariance matrix $E\left\{\boldsymbol{v} \boldsymbol{v}^{H}\right\}=\sigma^{2} \boldsymbol{I}$.

\section{BLOCK BIT ERROR RATE}

We define the block bit error rate, $P_{e}$, of the system in Fig. 1 to be the average bit error rate over all possible transmitted vectors, $P_{e}=E\left\{P_{e \mid s}\right\}$, where $P_{e \mid s}$ is the bit error rate for a given transmitted vector $\boldsymbol{s}$. For BPSK signals $P_{e}$ can be expressed as

$$
P_{e}=\frac{1}{M} \sum_{j=1}^{2^{M}} P_{s_{j}} \sum_{m=1}^{M} m P_{m \mid s_{j}},
$$

where $\boldsymbol{s}_{j}$ is the $j$ th element in the set of all possible antipodal vectors of length of $M, j \in\left[1,2^{M}\right] ; P_{m \mid s}$ is the probability of having $m$ erroneously detected bits given that $\boldsymbol{s}_{j}$ is the transmitted vector; and $P_{\boldsymbol{s}_{j}}$ is the probability that $\boldsymbol{s}_{j}$ is transmitted. It can be shown [3] that

$$
P_{e}=\frac{1}{2 M} \sum_{m=1}^{M} \operatorname{erfc}\left(\frac{1}{\sqrt{2 \sigma^{2}\left[\boldsymbol{G} \boldsymbol{G}^{H}\right]_{m m}}}\right),
$$




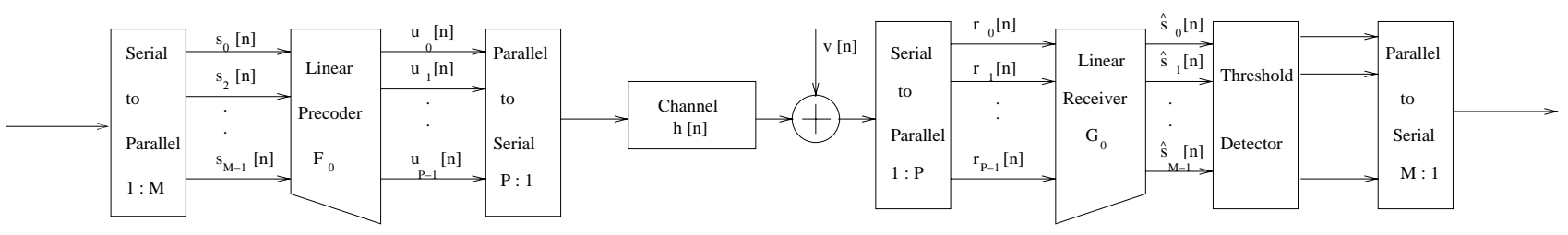

Fig. 1. A discrete-time baseband equivalent model of the block-by-block transceiver model developed in [8].

where $\operatorname{erfc}(z)=(2 / \sqrt{\pi}) \int_{z}^{\infty} \exp \left(-z^{2}\right) d z$.

A key element of our design technique is the observation that $P_{e}$ is a convex function of $\left[\boldsymbol{G} \boldsymbol{G}^{H}\right]_{\mathrm{mm}}$ when $\left[\boldsymbol{G} \boldsymbol{G}^{H}\right]_{\mathrm{mm}}$ is sufficiently small. To establish this fact, we define $\phi(x)=$ $\operatorname{erfc}\left(\frac{1}{\sqrt{2 \sigma^{2} x}}\right)$, for $x>0$. The second derivative of $\phi(x)$ is

$$
\frac{d^{2} \phi(x)}{d x^{2}}=\frac{1}{\sqrt{2 \pi \sigma^{2} x^{5}}}\left(\frac{1}{2 \sigma^{2} x}-\frac{3}{2}\right) \exp \left(-\frac{1}{2 \sigma^{2} x}\right)
$$

If $x<1 /\left(3 \sigma^{2}\right)$, then $d^{2} \phi(x) / d x^{2}>0$, and hence for those small values of $x, \phi(x)$ is a convex function.

\section{DESIGN OF THE MINIMUM BER PRECODER}

We seek to design an optimum precoder $\boldsymbol{F}$ such that the minimum BER is achieved subject to a bound on the transmitted power, $\operatorname{tr}\left(E\left\{\boldsymbol{F}_{0} \boldsymbol{s}\left(\boldsymbol{F}_{0} \boldsymbol{s}\right)^{H}\right\}\right)=\operatorname{tr}\left(\boldsymbol{F}_{0} \boldsymbol{F}_{0}^{H}\right)$. For ZP systems the total transmitted power is simply the power used to transmit the data, whereas for CP systems the total transmitted power is the sum of the power used to transmit the data and the power used to transmit the cyclic prefix. It is standard practice to define the transmitted power as simply the power required to transmit the data in $\mathrm{CP}$ systems $[1,5,9]$. (The extra power used to transmit the cyclic prefix is incorporated into some addition performance analysis in [3].) We can, therefore, formulate the design problem for both $\mathrm{ZP}$ and $\mathrm{CP}$ systems as follows:

$$
\begin{gathered}
\min _{\boldsymbol{F}} P_{e} \\
\text { subject to } \quad \operatorname{tr}\left(\boldsymbol{F} \boldsymbol{F}^{H}\right) \leq p_{0},
\end{gathered}
$$

where $p_{0}$ is the bound on the transmitted power. From Section 3, $P_{e}$ is a convex function of $\left[\boldsymbol{G} \boldsymbol{G}^{H}\right]_{m m}$ if $\left[\boldsymbol{G} \boldsymbol{G}^{H}\right]_{m m} \leq 1 /\left(3 \sigma^{2}\right)$, $\forall m \in[1, M]$. We will show in Section 5 that this condition can be interpreted as a sufficiently large signal-to-noise ratio. In the region in which $P_{e}$ is convex, we can apply Jensen's inequality [2] such that,

$$
\begin{aligned}
P_{e} & \geq \frac{1}{2} \operatorname{erfc}\left(\frac{1}{\sqrt{\frac{2 \sigma^{2}}{M} \sum_{m=1}^{M}\left[\boldsymbol{G} \boldsymbol{G}^{H}\right]_{m m}}}\right) \\
& =\frac{1}{2} \operatorname{erfc}\left(\frac{1}{\sqrt{2 \sigma^{2} \frac{\operatorname{tr}\left(\boldsymbol{G G} \boldsymbol{G}^{H}\right)}{M}}}\right) \triangleq P_{e, \mathrm{LB}} .
\end{aligned}
$$

Equality in (6) holds if and only if $\left[\boldsymbol{G} \boldsymbol{G}^{H}\right]_{m m}$ are equal, $\forall m \in$ $[1, M]$. Since $P_{e, \mathrm{LB}}$ in (7) defines a lower bound on the BER, an optimum precoder can be obtained via the following two stages:
- Stage 1: Minimize the lower bound, $P_{e, \mathrm{LB}}$, subject to the constraint on transmitted power and the condition that $P_{e}$ remains convex.

- Stage 2: Show that a particular choice of $\boldsymbol{F}$ from the solution set for Stage 1 achieves the minimized lower bound.

To perform Stage 1, we note that $\operatorname{erfc}(\cdot)$ is a monotonically decreasing function. Thus, to minimize $P_{e, \mathrm{LB}}$ in (7) we need only minimize $\operatorname{tr}\left(\boldsymbol{G} \boldsymbol{G}^{H}\right)$. The zero-forcing equalizer for the system in (1) is $\boldsymbol{G}=(\boldsymbol{H} \boldsymbol{F})^{\dagger}$, where $(\cdot)^{\dagger}$ denotes the Moore-Penrose pseudo inverse. In the ZP case $\boldsymbol{H}$ has full column rank for all $L$ th order channels except the identically zero channel, and in the $\mathrm{CP}$ case $\boldsymbol{H}$ has full rank unless the channel has a zero exactly on the DFT grid. (In practice, any such sub-channels would be 'dropped' using the procedure in Section 5.) Therefore, since $\boldsymbol{F}$ is square, we can almost always write $\boldsymbol{G}=\boldsymbol{F}^{-1} \boldsymbol{H}^{H}\left(\boldsymbol{H}^{H} \boldsymbol{H}\right)^{-1}$. To complete Stage 1 we must solve

$$
\begin{array}{ll} 
& \min _{\boldsymbol{F}} \operatorname{tr}\left(\boldsymbol{F}^{-1}\left(\boldsymbol{H}^{H} \boldsymbol{H}\right)^{-1} \boldsymbol{F}^{-H}\right) \\
\text { subject to } & \operatorname{tr}\left(\boldsymbol{F} \boldsymbol{F}^{H}\right) \leq p_{0} \\
& {\left[\boldsymbol{F}^{-1}\left(\boldsymbol{H}^{H} \boldsymbol{H}\right)^{-1} \boldsymbol{F}^{-H}\right]_{m m} \leq \frac{1}{3 \sigma^{2}}, \quad \forall m \in[1, M],}
\end{array}
$$

Problem (8) is not convex in $\boldsymbol{F}$ and hence can be awkward to solve directly. However, if we relax (8) by dropping (8c), then the problem can be made convex in $\boldsymbol{R}=\boldsymbol{F} \boldsymbol{F}^{H}$. In fact, that convex relaxation can be solved analytically, and the set of solutions is [8]

$$
\boldsymbol{F}_{\text {rel }}=\sqrt{\frac{p_{0}}{\operatorname{tr}\left(\boldsymbol{\Lambda}^{1 / 2}\right)}} \boldsymbol{W} \boldsymbol{\Lambda}^{1 / 4} \boldsymbol{U}
$$

where $\left(\boldsymbol{H}^{H} \boldsymbol{H}\right)^{-1}=\boldsymbol{W} \boldsymbol{\Lambda} \boldsymbol{W}^{H}$ is an eigen decomposition of $\left(\boldsymbol{H}^{H} \boldsymbol{H}\right)^{-1}$, and $\boldsymbol{U}$ is an arbitrary unitary matrix. To complete Stage 1, we will use (9) and the following lemma to generate a solution to (8).

Lemma 1 ( [3]). Given an $M \times M$ positive semidefinite matrix $\boldsymbol{S}$ with eigen decomposition $\boldsymbol{V} \boldsymbol{\Gamma} \boldsymbol{V}^{H}$,

$$
\operatorname{tr}(\boldsymbol{S})=M \tau \Longleftrightarrow\left[\boldsymbol{D}^{H} \boldsymbol{V}^{H} \boldsymbol{S} \boldsymbol{V} \boldsymbol{D}\right]_{m m}=\tau, \quad \forall m \in[1, M],
$$

where $\boldsymbol{D}$ is the normalized $M \times M$ DFT matrix.

Using Lemma 1, we have that: If $\left(\operatorname{tr}\left(\boldsymbol{\Lambda}^{1 / 2}\right)\right)^{2} / p_{0}>$ $M /\left(3 \sigma^{2}\right)$, the feasible set for the constraint in (8c) is empty, and hence there is no solution for (8). On the other hand, if

$$
\frac{\left(\operatorname{tr}\left(\boldsymbol{\Lambda}^{1 / 2}\right)\right)^{2}}{p_{0}} \leq \frac{M}{3 \sigma^{2}},
$$


then there is at least one solution to (8). In particular, one precoder $\boldsymbol{F}$ which minimizes $P_{e, \mathrm{LB}}$ and hence solves Stage 1 is

$$
\boldsymbol{F}_{\mathrm{opt}}=\sqrt{\frac{p_{0}}{\operatorname{tr}\left(\boldsymbol{\Lambda}^{1 / 2}\right)}} \boldsymbol{W} \boldsymbol{\Lambda}^{1 / 4} \boldsymbol{D} .
$$

(If $M$ is a power of 2, the normalized Hadamard matrix can be used in place of $\boldsymbol{D}$ in Lemma 1 and (11), if so desired.)

To complete Stage 2 and hence determine a minimum BER precoder, we simply observe that $\boldsymbol{F}_{\text {opt }}$ in (11) makes all $\left[\boldsymbol{G} \boldsymbol{G}^{H}\right]_{m m}$ equal [3]. Hence, $\boldsymbol{F}_{\text {opt }}$ not only minimizes the lower bound on the BER, it actually achieves that minimized lower bound. Therefore, $\boldsymbol{F}_{\text {opt }}$ is a precoder which minimizes the BER.

The relaxed design problem consisting of (8a) and (8b) can be interpreted as the minimization of the mean square error of the data estimates, subject to zero-forcing equalization and a transmitted power constraint; i.e., $\boldsymbol{F}_{\text {MMSE }}=\boldsymbol{F}_{\text {rel }}$. The solution set for this problem [c.f., (9)] is parameterized by a unitary matrix. All unitary matrices produces the same (minimized) MSE, but the BER is dependent on the choice of the unitary matrix. In other words, the minimum BER (MBER) precoder is a special MMSE precoder with a carefully chosen degree of freedom, but MMSE precoders do not necessarily provide minimum BER.

\section{SUB-CHANNEL DROPPING SCHEME}

If we define the signal to noise ratio (SNR) as the ratio of the transmitted power per block to the receiver noise power per block, $\rho \triangleq p_{0} /\left(P \sigma^{2}\right)$, the condition in (10) for the existence of solutions to $(8)$ can be expressed as

$$
\rho \geq \frac{3\left(\operatorname{tr}\left(\boldsymbol{\Lambda}^{1 / 2}\right)\right)^{2}}{P M} \triangleq \rho_{c},
$$

which indicates that our MBER precoder is valid at moderate-tohigh SNRs. This condition can be ensured by either increasing the transmitted power or by dropping the sub-channels corresponding to the largest values in $\boldsymbol{\Lambda}$. Dropping sub-channels corresponds to avoiding transmission on the low-gain sub-channels, and reallocating transmission power among the surviving ones. The benefit of sub-channel dropping is that a minimum BER precoder can be guaranteed without violating the transmission power budget. However, the block size becomes smaller, and therefore the transmission rate is lower (if the bit loading is uniform). The design of an MBER precoder with sub-channel dropping is as follows:

- Suppose that $\Lambda$ is ordered in descending order and let $\bar{M}=$ $M, \bar{\Lambda}=\Lambda$

- First determine the new block size $\bar{M}$ :

While $\rho<3\left(\operatorname{tr}\left(\bar{\Lambda}^{1 / 2}\right)\right)^{2} /(P \bar{M})$, set $\bar{M}=\bar{M}-1$, and set the largest element in $\bar{\Lambda}$ to zero.

- Then calculate the MBER precoder $\boldsymbol{F}_{\text {MBER-DROP }}$ after dropping sub-channels:

Form $\overline{\boldsymbol{F}}_{\text {DROP }}$ by removing the first $M-\bar{M}$ columns from the matrix $\sqrt{\frac{p_{0}}{\operatorname{tr}\left(\overline{\boldsymbol{\Lambda}}^{1 / 2}\right)}} \boldsymbol{W} \overline{\boldsymbol{\Lambda}}^{1 / 4}$. Then $\boldsymbol{F}_{\text {MBER-DROP }}=$ $\overline{\boldsymbol{F}}_{\text {DROP }} \overline{\boldsymbol{D}}$, where $\overline{\boldsymbol{D}}$ is a normalized $\bar{M} \times \bar{M}$ DFT matrix.

The resulting optimal precoder $\boldsymbol{F}_{\text {MBER-DROP }}$ is of size of $M \times \bar{M}$ (i.e., a tall matrix), implying that of the $M$ sub-channels, $M-$ $\bar{M}+1$ sub-channels are dropped.

\section{MBER PRECODERS FOR CP SYSTEMS}

From the derivation in Section 4, the ZP-MBER and CP-MBER precoders have the same general formula [c.f., (11)], but the matrices $\boldsymbol{W}$ and $\boldsymbol{\Lambda}$ are different due to the different channel matrices for ZP and CP schemes [c.f., (1)]. Since the channel matrix for CP is circulant, it can be diagonalized by DFT and IDFT matrices, and therefore the MBER precoder for CP systems can be re-written as

$$
\boldsymbol{F}_{\mathrm{CP}-\mathrm{MBER}}=\alpha_{c p} \boldsymbol{D}^{H}\left(\boldsymbol{\Delta}_{H}^{-1} \boldsymbol{\Delta}_{H}^{-H}\right)^{1 / 4} \boldsymbol{D},
$$

where $\alpha_{c p}=\sqrt{\frac{p_{0}}{t r\left(\left(\boldsymbol{\Delta}_{H}^{-1} \boldsymbol{\Delta}_{H}^{-H}\right)^{1 / 2}\right)}}$, and $\boldsymbol{\Delta}_{H}$ is a diagonal matrix of sub-channel gains with $m$ th diagonal element $H\left(e^{j 2 \pi(m-1) / M}\right)=\sum_{n} h[n] e^{-j 2 \pi(m-1) n / M}, m \in[1, M]$. Similarly, the set of MMSE precoders for CP systems can be extracted from (9) as

$$
\boldsymbol{F}_{\mathrm{CP}-\mathrm{MMSE}}=\boldsymbol{D}^{H} \boldsymbol{\Delta}_{\mathrm{MMSE}} \boldsymbol{U}
$$

where $\boldsymbol{\Delta}_{\mathrm{MMSE}}=\alpha_{c p}\left(\boldsymbol{\Delta}_{H}^{-1} \boldsymbol{\Delta}_{H}^{-H}\right)^{1 / 4}$ is the MMSE power loading matrix, and $\boldsymbol{U}$ is an arbitrary unitary matrix. Therefore, the CPMBER precoder can be written as

$$
\boldsymbol{F}_{\mathrm{CP}-\mathrm{MBER}}=\boldsymbol{D}^{H} \boldsymbol{\Delta}_{\mathrm{MMSE}} \boldsymbol{D},
$$

which indicates that the CP-MBER precoder is related to standard DMT in that the diagonal power loading matrix in standard DMT is replaced by a full matrix which consists of a diagonal MMSE power loading matrix post-multiplied by a DFT matrix.

\section{SIMULATION RESULTS}

We now evaluate the BER performance of various $\mathrm{ZP}$ and $\mathrm{CP}$ precoders. The transmitted power is normalized (i.e., $p_{0}=1$ ) and the third-order FIR channel has zeros at $0.7,0.5 \exp (j 2 \pi 0.256)$, and $0.3 \exp (j 2 \pi 0.141)$. The block sizes are $M=32$ and $P=$ $M+L=35$. BER curves for various $\mathrm{ZP}$ and $\mathrm{CP}$ precoders are presented in Fig. 2. In Fig. 2(a) we compare the BER performance of ZP precoders designed using the MBER, MMSE [8], and maximum SNR [8] criteria, and a zero-padded OFDM [6] precoder In Fig. 2(b) we compare the BER performance of CP transmission schemes designed using the MBER, MMSE and water-filling DMT $[1,5]$ criteria, and a conventional OFDM scheme. In both the $\mathrm{ZP}$ and $\mathrm{CP}$ cases we show the performance of the MMSE precoder where the unitary matrix degree of freedom is the identity. To ensure a fair comparison between water-filling DMT and the MBER precoders, Figs 2(b) and (c) also contain BER curves for MBER precoders with the same block size as the water-filling DMT system. (Performance comparisons for the sub-channel dropping scheme in Section 5 are available in [3].) Figs 2(a) and (b) demonstrate the optimality of the MBER designs at moderate-to-high SNRs [c.f., (12)], and Fig. 2(c) shows the (small) performance advantage of ZP-MBER precoding over CP-MBER precoding. (The advantage is actually slightly larger than shown, as we have ignored the power used to transmit the cyclic prefix in our definition of SNR; see [3] for more details.) However, ZP-MBER precoding requires the calculation of the eigen-vectors of $\left(\boldsymbol{H}^{H} \boldsymbol{H}\right)^{-1}$ for each different channel, whereas for the $\mathrm{CP}$ precoder the eigenvectors of $\left(\boldsymbol{H}^{H} \boldsymbol{H}\right)^{-1}$ are merely the columns of an IDFT matrix, irrespective of the actual channel coefficients. Furthermore, equalization of the CP-MBER scheme can be implemented more efficiently than that of the ZP-MBER scheme. 


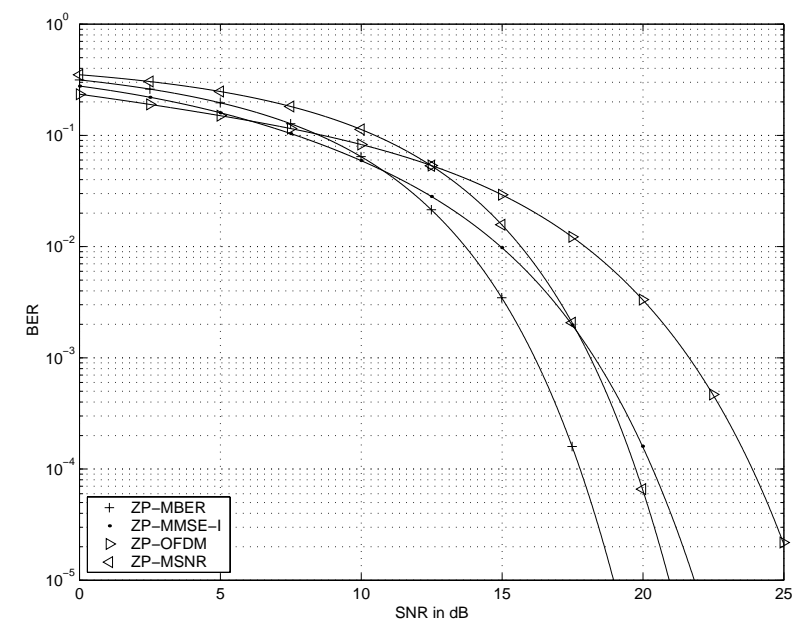

(a) ZP Precoders

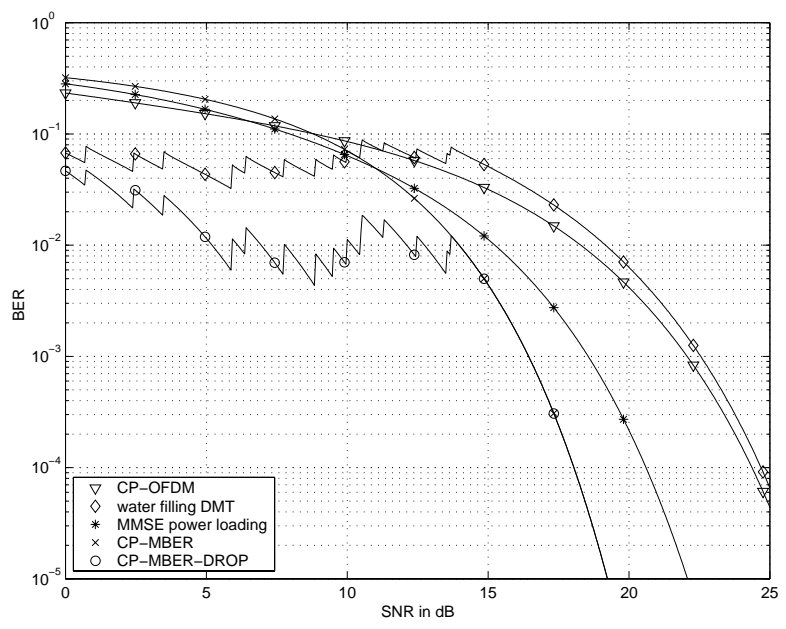

(b) CP Precoders

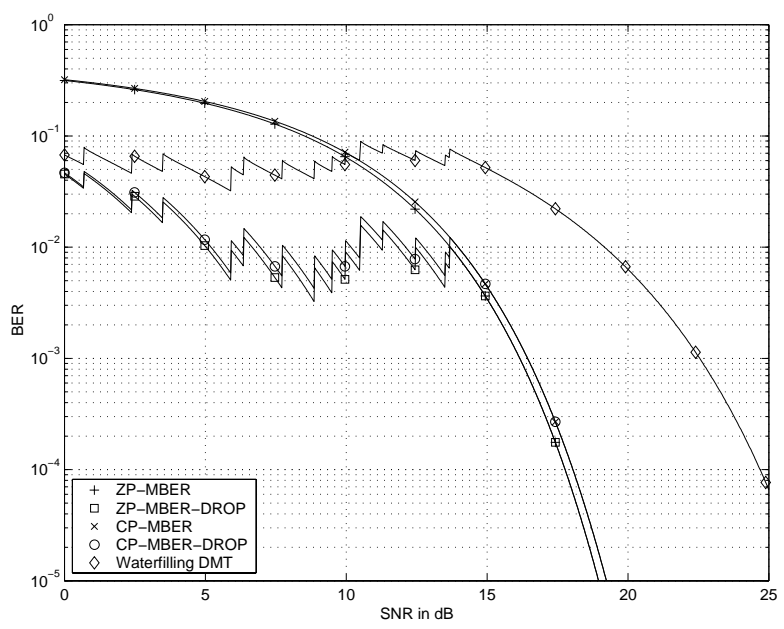

(c) ZP and CP MBER precoders and water-filling DMT

Fig. 2. BER curves for $\mathrm{ZP}$ and $\mathrm{CP}$ precoders.

\section{CONCLUSION}

In this paper, the linear precoder which minimizes the bit error rate (BER) was derived for block transmission systems in which zero-forcing equalization and threshold detection are applied. Analytic solutions for the optimal precoder were derived for two standard schemes of eliminating inter-block interference (namely, zero-padded and cyclic-prefix schemes), and a flexible scheme for dropping channels to ensure the validity of the analytic solution was proposed. It was found that the $\mathrm{CP}$ transmission scheme with the MBER precoding can be regarded as variation on standard DMT in which MMSE power loading replaces water-filling power loading, and the diagonal power loading matrix is post-multiplied by a DFT matrix. Performance evaluations indicate that the SNR gain of the MBER design over some conventional designs may be as much as several decibels. Thus, the MBER design proposed in this paper is an attractive alternative for realizing linear precoders for block-by-block data transmission with zero-forcing equalization.

The optimal design obtained in this paper is for a single-user system with zero-forcing equalization, white data symbols from a simple constellation, and a known channel. Continuing work in this area includes extensions to multiuser systems, other equalization techniques (such as MMSE, decision feedback, or maximum likelihood detection), colored data from higher-order constellations, and uncertain channel models.

\section{REFERENCES}

[1] J. S. Chow, J. C. Tu and J. M. Cioffi, "Performance evaluation of a multichannel transceiver system for ADSL and VHDSL receivers," IEEE J. Select. Areas Commun., vol. 9, pp. 909-919, Aug. 1991.

[2] T. M. Cover and J. A. Thomas, Elements of Information Theory. Wiley, 1991.

[3] Y. W. Ding, "Minimum BER block precoders for zeroforcing equalization", M. Eng. Thesis, McMaster University, Hamilton, Ontario, Canada, Oct. 2001.

[4] N. J. Fliege, "Orthogonal multiple carrier data transmission," Euro. Trans. Telecommun., vol. 3, pp. 255-264, May 1992.

[5] B. S. Krongold, K. Ramchandran, and D. L. Jones, "Computationally efficient optimal power allocation algorithms for multicarrier communication systems," IEEE Trans. Commun., vol. 48, pp. 23-27, Jan. 2000.

[6] B. Muquet, M. de Courville, G. B. Giannakis, Z. Wang, and P. Duhamel, "Reduced complexity equalizers for zeropadded OFDM transmissions," in Proc. Int. Conf. Acoust. Speech, Signal Processing, pp. 2973-2976, Istanbul, Turkey, June 2000.

[7] A. Scaglione, S. Barbarossa, and G. B. Giannakis, "Filterbank tranceivers optimizing information rate in block transmissions over dispersive channels," IEEE Trans. Inform. Theory, vol. 45, pp. 1988-2006, Apr. 1999.

[8] A. Scaglione, G. B. Giannakis, and S. Barbarossa, "Redundant filterbank precoders and equalizers, Parts I and II," IEEE Trans. Signal Processing, vol. 47, pp. 1988-2022, July 1999.

[9] Z. Wang and G. B. Giannakis, "Wireless multicarrier communications," IEEE Signal Processing Mag., pp. 29-48, May 2000 . 